\title{
Comparison of methods for the implementation of genome-assisted evaluation of Spanish dairy cattle
}

\author{
J. A. Jiménez-Montero, ${ }^{* 1}$ O. González-Recio,† and R. Alenda* \\ *Departamento de Producción Animal, Escuela Técnica Superior de Ingenieros (ETSI) Agrónomos-Universidad Politécnica de Madrid, \\ 28040 Madrid, Spain \\ †Departamento de Mejora Genética Animal, Instituno Nacional de Investigación y Tecnología Agraria y Alimentaria (INIA), 28040 Madrid, Spain
}

\begin{abstract}
The aim of this study was to evaluate methods for genomic evaluation of the Spanish Holstein population as an initial step toward the implementation of routine genomic evaluations. This study provides a description of the population structure of progeny tested bulls in Spain at the genomic level and compares different genomic evaluation methods with regard to accuracy and bias. Two Bayesian linear regression models, Bayes-A and Bayesian-LASSO (B-LASSO), as well as a machine learning algorithm, Random-Boosting (R-Boost), and BLUP using a realized genomic relationship matrix (GBLUP), were compared. Five traits that are currently under selection in the Spanish Holstein population were used: milk yield, fat yield, protein yield, fat percentage, and udder depth. In total, genotypes from 1859 progeny tested bulls were used. The training sets were composed of bulls born before 2005; including 1601 bulls for production and 1574 bulls for type, whereas the testing sets contained 258 and 235 bulls born in 2005 or later for production and type, respectively. Deregressed proofs (DRP) from January 2009 Interbull (Uppsala, Sweden) evaluation were used as the dependent variables for bulls in the training sets, whereas DRP from the December 2011 DRPs Interbull evaluation were used to compare genomic predictions with progeny test results for bulls in the testing set. Genomic predictions were more accurate than traditional pedigree indices for predicting future progeny test results of young bulls. The gain in accuracy, due to inclusion of genomic data varied by trait and ranged from 0.04 to 0.42 Pearson correlation units. Results averaged across traits showed that B-LASSO had the highest accuracy with an advantage of 0.01, 0.03 and 0.03 points in Pearson correlation compared with R-Boost, Bayes-A, and G-BLUP, respectively. The B-LASSO predictions also showed the least bias (0.02, 0.03 and 0.10 SD units less than Bayes-
\end{abstract}

Received April 17, 2012

Accepted September 17, 2012.

${ }^{1}$ Corresponding author: joseantonio.jimenez.montero@upm.es
A, R-Boost and G-BLUP, respectively) as measured by mean difference between genomic predictions and progeny test results. The R-Boosting algorithm provided genomic predictions with regression coefficients closer to unity, which is an alternative measure of bias, for 4 out of 5 traits and also resulted in mean squared errors estimates that were $2 \%, 10 \%$, and $12 \%$ smaller than B-LASSO, Bayes-A, and G-BLUP, respectively. The observed prediction accuracy obtained with these methods was within the range of values expected for a population of similar size, suggesting that the prediction method and reference population described herein are appropriate for implementation of routine genomeassisted evaluations in Spanish dairy cattle. R-Boost is a competitive marker regression methodology in terms of predictive ability that can accommodate large data sets.

Key words: genome-assisted evaluation, machine learning, dairy cattle, predictive ability

\section{INTRODUCTION}

Genomic selection (GS) is the most promising new technology since progeny testing to increase the rate of genetic gain in dairy cattle (Weigel et al., 2010). It is based on simultaneous selection for thousands of SNP. Direct genomic breeding values (DGV) can be calculated as the sum of the effects of individual SNP across the entire genome, or genome-enhanced predictions can be computed by augmentation or by replacing the traditional pedigree relationship matrix with the realized genomic matrix (Goddard, 2009). Typically, SNP effects are first estimated in a training or reference population and then used to predict the breeding values of new selection candidates (Hayes et al., 2009a).

In dairy cattle, GS has profoundly changed practical breeding programs, because nearly all young bulls acquired by major AI centers are now selected based on such evaluations (Wiggans et al., 2011). In addition, females can be evaluated with cost-effective genotyping strategies (Weigel et al., 2012), leading to genomic predictions with a similar reliability to that of young bulls. 
National and international genetic evaluations of dairy cattle consider nearly 2 dozen phenotypic traits (VanRaden and Sullivan, 2010) and the inclusion of additional, complex traits is expected within the next decade. These new traits may include measures of disease resistance and residual feed intake (González-Recio and Forni, 2011; Pryce et al., 2012), and evaluation may consider crossbreed performance (Toosi et al., 2010), and genotype by environment interaction effects (Hayes et al., 2009b).

Several different approaches are currently used for estimating genomic values, and it is important to assess the performance of diverse methodologies and identify the methods that can provide the greatest predictive accuracy in a given population. Genomic prediction methods can be categorized as (1) methods that regress phenotypic records on SNP markers directly, and (2) methods that view genetic values as a function of the subject and use marker information to build the (co)variance structure between subjects (de los Campos et al., 2009). The first group of methods includes several Bayesian regression approaches, such as Bayes-A, Bayes-B (Meuwissen et al., 2001), and the Bayesian least absolute shrinkage selection operator (B-LASSO), as described by Park and Casella (2008). These regression-based methods are usually implemented after traditional BLUP genetic evaluation of the reference population, and the resulting breeding value estimates are then used directly or deregressed before use as a dependent variable in the genomic evaluation (VanRaden, 2008). In general, these methods are computationally time consuming if the number of SNP is large, which could preclude their utilization in routine evaluation programs in some countries, despite the fact that they are currently used in some countries, such as the Netherlands. The second group includes methods that compute a realized relationship matrix from the markers, such as genomic (G)-BLUP (VanRaden et al., 2009) or single-step (Misztal et al., 2009) methods, to augment or replace the traditional pedigree based relationship matrix. The single-step method includes all pedigree and genomic information and avoids the need to subsequently combine the genomic and traditional breeding values (Aguilar et al., 2010). The performance of alternative genomic evaluation methodologies can vary depending on the trait and population structure (Daetwyler et al., 2010).

In addition to the aforementioned approaches, an alternative for dealing with large data sets and complex interactions between SNP is machine learning algorithms (Long et al., 2007). Machine learning methods usually compare favorably to Bayesian regression models in terms of predictive ability (e.g., González-Recio et al., 2008; Moser et al., 2009; González-Recio and Forni, 2011). Nonparametric or semiparametric methods of this type can be implemented by regressions on markers (e.g., boosting, as in González-Recio et al., 2010) or by building appropriate (co)variance structures (e.g., reproducing kernel Hilbert spaces regression; Gianola and van Kaam, 2008). Boosting algorithms are among the most appealing machine learning methods for genomic prediction problems (Ogutu et al., 2011) and in a recent study they provided greater predictive ability and smaller bias than other methods (González-Recio et al., 2010). Efficiency of DGV prediction in dairy cattle can be enhanced by modification to the algorithm, specifically random boosting (R-Boost), as described in the companion paper (González-Recio et al., 2013). These modifications allow prediction of genomic values with SNP regression methods in very large data sets.

Over the last decade, the Spanish breeding program has provided competitive bulls for the national and international markets due to a robust milk-recording scheme. Special care has been taken in recording morphologic traits. Genomic selection has revolutionized dairy cattle breeding since 2009. Taking advantage of this technology is necessary to maintain the program's viability.

The objective of this study was to use genotypic and phenotypic data from the Spanish Holstein population to compare several popular genomic evaluation methodologies. Two different Bayesian linear regressions (Bayes-A and B-LASSO), G-BLUP, and a machine learning algorithm (R-Boost) were compared. Five phenotypic traits were considered, and methods were evaluated based on predictive correlations, bias, and mean squared error.

\section{MATERIALS AND METHODS}

\section{Genotypes}

A total of 1,859 progeny-tested sires were genotyped. The BovineSNP50.v2 Beadchip (Illumina Inc., San Diego, CA) was used to genotype 54,609 SNP of 1,619 sires, and the remaining 240 sires were genotyped for 54,001 SNP using the BovineSNP50.v1 Beadchip (Illumina Inc.).

Single nucleotide polymorphisms with a greater than 5\% incidence of missing genotypes across individuals and SNP with minor allele frequency (MAF) less than $5 \%$ were discarded, leaving only 39,714 SNP for analysis. Some animals had missing genotypes for certain markers; after editing, $0.01 \%$ of the SNP were missing. Missing genotypes were then imputed with Beagle 3.3.2 (Browning and Browning, 2009). In a pilot 
study, known SNP were masked, mimicking the missing marker rate of the population. Resulted imputation allele error ratio was 0.008 .

\section{Linkage Disequilibrium Estimation}

The haplotypes obtained by Beagle prior imputation were used to estimate the degree of linkage disequilibrium (LD) between SNP; all genotyped bulls were used in this calculation. Linkage disequilibrium, which refers to the nonrandom association of alleles between 2 loci, was measured using the $r^{2}$ parameter (Hill and Robertson, 1968). Linkage disequilibrium can be estimated using other methods, such as $D, D^{\prime}$ or measures based on the Chi-squared statistic (Zhao et al., 2005); however, $r^{2}$ is the most common measure of LD for biallelic markers and is less sensitive to the effects of allelic frequency than other methods (Sargolzaei et al., 2008).

\section{Phenotypes}

The January 2009 deregressed multiple across-country evaluation (MACE) proofs (DRP) from progeny testing, as described in Jairath et al. (1998), were used as dependent variables and included 1,859 bulls for production and 1,810 for type. The production and type data were collected from progeny between 1980 and 2008. Sire DRP for milk yield (MY), fat yield $(\mathbf{F Y})$, protein yield $(\mathbf{P Y})$, fat percentage $(\mathbf{F P})$, and udder depth (UD) were used. The estimated heritability based on traditional genetic evaluations in Spain is 0.28 and 0.30 for production traits and udder depth, respectively.

\section{Training and Validation Data Sets}

Training and validation data sets were generated based on year of birth of the bulls. A total of 1,601 bulls with DRP in January 2009 that were born before 2005 were used for the production training set, whereas 1,574 bulls from the same period were used for the type training set. Bulls born between 2005 and 2007 were used as the validation set; 258 bulls were used for production traits and 236 were used for type. Effective daughter contributions (EDC) were used as weighting factors to account for differences in progeny group size when computing genomic predictions (Jairath et al., 1998). Bulls in the testing sets had their DGV in December 2011 that were based on 20 or more EDC. Design of the training and testing sets followed the recommendations of Mäntysaari et al. (2010), although the recommended 4-yr gap between training and test- ing sets was reduced to $3 \mathrm{yr}$ because of the small size of the reference population, thereby leaving more training set bulls to maximize the accuracy of estimated DGV.

\section{Genomic Evaluation Model}

The general structure for the models in linear form is

$$
\mathbf{y}=\mu \mathbf{1}_{n}+\sum_{j} \mathbf{X}_{j} g_{j}+\mathbf{e}
$$

where $\mathbf{y}$ is the vector of phenotypic records, $\mu$ is the overall mean, $\mathbf{1}_{n}$ is a vector of $n$ ones, $\Sigma_{j}$ is a summation over all markers, $g_{j}$ is the coefficient of marker $j$ denoting the allele substitution effect, $\mathbf{X}_{j}$ is a design matrix of genotype codes for the respective marker, and $\mathbf{e}$ is a vector of residuals.

The data were analyzed using 4 different approaches: 2 models based on marker regression (Bayes-A and B-LASSO), a method based on a realized relationship matrix from the markers (G-BLUP), and a machine learning algorithm (R-Boost), which is described in the companion paper (González-Recio et al., 2013).

\section{Bayes-A}

Bayes-A was introduced by Meuwissen et al. (2001). This method assumes that marker effects $\left(g_{j}\right)$ are normally and independently distributed a priori as $N=\left(0, \sigma_{g_{j}}^{2}\right)$, where $\sigma_{g_{j}}^{2}$ is an unknown variance associated with marker $j$. The prior distribution of the variances of the SNP was a scaled inverted chi-squared distribution, $\sigma_{j}^{2} \sim \chi_{(d f, s s)}^{-2}$, where $s s$ is the scale parameter and $d f$ represents the degrees of freedom. The parameters $s s$ and $d f$ were considered as hyper-parameters and were fixed for each trait as in Gianola et al. (2009). An improper prior was assumed for $\mu$. Following González-Recio et al. (2009), the residuals, $e$, were assumed to be distributed as $N\left(0, R=N^{-1} \sigma_{e}^{2}\right)$, where $\mathbf{N}$ $=\left\{n_{i}\right\}$ is a diagonal matrix with elements $n_{i}$ representing the corresponding EDC of sire $i$, and $\sigma_{e}^{2}$ is the residual variance. The prior distribution for the residual variance $\sigma_{e}^{2}$ was assumed to be an inverted chi-squared distribution with hyper-parameters $d f$ and ss. The Gibbs sampler was run for 10,000 cycles, with the first 1,000 cycles of burn-in discarded. Convergence of the chain was checked by visual inspection, and inferences on the parameters were made on the mean posterior estimates after burn-in. 


\section{Bayesian-LASSO}

The Bayesian counterpart of the LASSO model (Park and Casella, 2008; de los Campos et al., 2009) was also used to estimate SNP coefficients in the training population. The B-LASSO model can be viewed as an optimization problem, using the sum of the absolute values of the regression coefficients ( $L 1$-norm) as a penalty, in the following regression model (Tibshirani, 1996):

$$
\min _{\beta}\left\{\sum\left(\mathrm{y}_{j}-\mathbf{X}_{j}^{\prime} \boldsymbol{\beta}\right)^{2}+\lambda(t) \sum_{j}\left|\boldsymbol{\beta}_{j}\right|\right\},
$$

where $\mathbf{X}$ is a vector of covariates, $\boldsymbol{\beta}$ is the corresponding vector of regression coefficients, $\lambda$ is a smoothing parameter controlling the shrinkage of the distribution, and $t$ is an arbitrary positive constant.

The LASSO estimates can be interpreted as the posterior mode in a Bayesian model considering a double Laplace prior for the coefficient estimates, as follows:

$$
\pi(\boldsymbol{\beta})=\prod_{j=1}^{p} \frac{\lambda}{2} e^{-\lambda\left|\beta_{j}\right|}
$$

as put forth by Park and Casella (2008), the smoothing parameter $\lambda$ was assigned a prior distribution gamma (a, b), $p=$ independent, zero-mean, double-exponential (DE) densities, and $e=$ exp. Values of the hyperparameters of the prior distribution were set at 5.0 and 1.0 , respectively, for convenience.

The Laplace distribution results in stronger shrinkage of marker coefficients toward zero than Bayes-A. This prior gives each coefficient $\beta_{j}$ a high probability of being near zero while simultaneously giving some coefficients a chance to have large effect ( $\mathrm{Yi}$ and $\mathrm{Xu}, 2008$ ). In practice, this produces a similar outcome to variable selection (de los Campos et al., 2010). Flat prior was assumed for $\mu$. The prior distribution for the residual variance, $\sigma_{e}^{2}$, was assumed to be an inverted chi-squared distribution with hyper-parameters $d f$ and $s s$ and was weighted by the number of progeny of each bull, as detailed for the previous method. A single chain of Gibbs sampling was run using 25,000 iterations and a burn-in period of 15,000 . The convergence of the chain was checked by visual inspection, and inferences on the parameters were made on the mean posterior estimates after burn-in.

\section{Genomic BLUP}

The G-BLUP is the most similar to traditional BLUP evaluations of the 4 alternatives considered herein. If many QTL exist with effects that are normally distributed with constant variance, the pedigree relationship matrix can be replaced by the genomic relationship matrix $(\mathbf{G})$, where the latter is built from molecular information. Pairs of individuals sharing the same genotype for a large number of markers will be more similar genomically and will have higher values in the corresponding off-diagonal cells of the matrix, as is the case for pairs of related animals in a pedigree-based relationship matrix. The genomic relationship matrix was computed as

$$
\mathbf{G}=\frac{\mathbf{Z Z}^{\prime}}{2 \sum p_{i}\left(1-p_{i}\right)}
$$

where $p$ is the frequency of the second allele and $i$ is the locus, $\mathbf{Z}$ is a matrix that results from the subtraction of $\mathrm{P}$ from $\mathbf{M}$, being $\mathrm{P}=2\left(p_{i}-0.5\right)$, and $\mathbf{M}$ the matrix of genotypes codified as $-1,0$, and 1 for the homozygote, heterozygote, and other homozygote, respectively, following VanRaden (2008), where a more detailed description of this model is provided.

\section{Random Boosting}

The R-Boost algorithm is a machine learning technique that combines several different predictors and a shrinkage factor (Friedman, 2001). Boosting iteratively adds basis functions, such that each addition further reduces the selected loss function (Hastie et al., 2009). In this study, ordinary least squares was chosen as the basis function, and it was successively applied to the residuals of the previous iteration in a sequential manner. The mean squared error (MSE) of prediction was used as the loss function to be minimized. Details of the algorithm are described in the companion paper (González-Recio et al., 2013). Following their results, $v$ (a shrinkage parameter) was set to 0.10 for production traits and 0.20 for type, and the percentage of SNP selected at each iteration ( $\boldsymbol{m t r y}$ ) was set to 0.50, 0.10, $0.01,1.00$, and 0.10 for MY, FY, PY, FP, and UD, respectively.

\section{Estimation of DGV}

The DGV for each trait were calculated for individuals in the testing set as follows:

$$
D G V=\mu+\mathbf{X}_{j} \hat{\boldsymbol{\beta}}_{j}
$$

where $\mu$ is the overall mean, $\mathbf{X}_{j}$ is a matrix of genotypes, and $\hat{\boldsymbol{\beta}}_{j}$ is a vector of posterior means of SNP ef- 


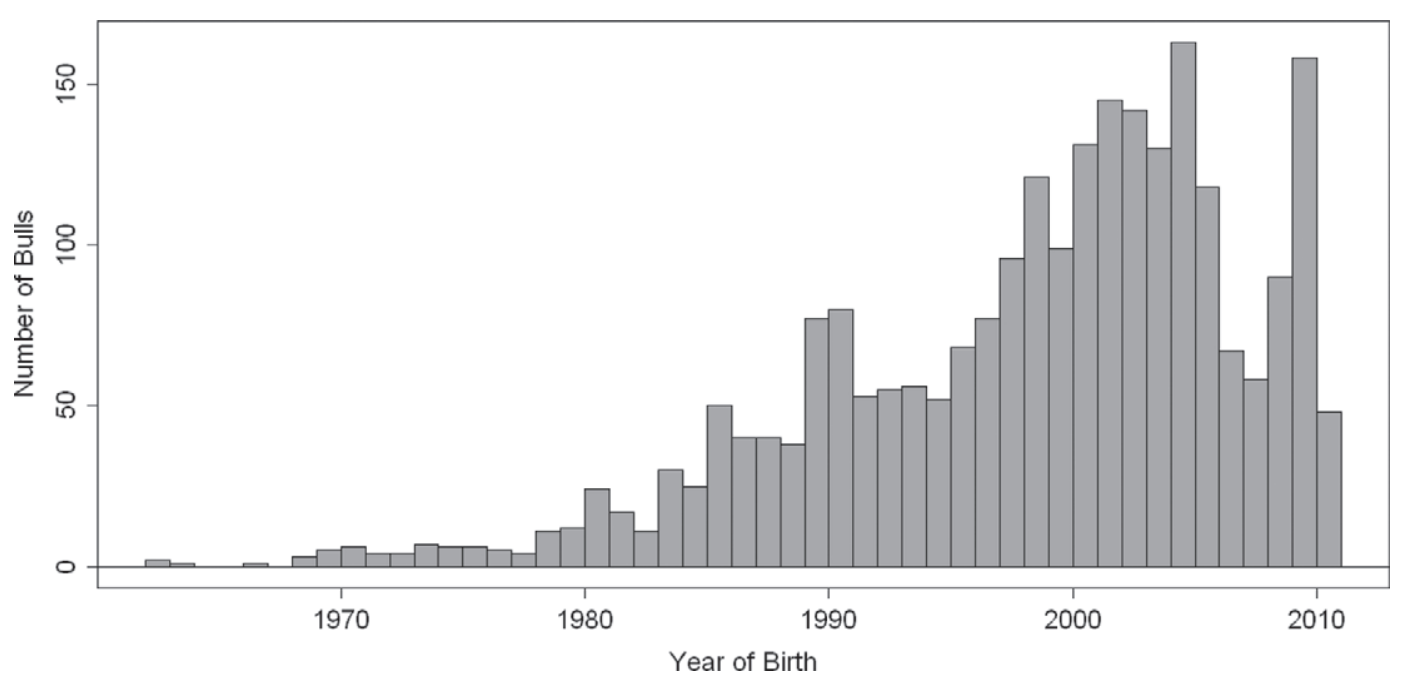

Figure 1. Number of genotyped bulls by year of birth.

fects for each of the 4 methods. For the R-Boost method, $\hat{\boldsymbol{\beta}}_{j}$ represents the sum of the slope estimates from the model in which SNP $j$ was selected.

\section{Criterion for Comparisons}

The accuracy of the genomic predictions was computed as the Pearson correlation between the predicted DGV of bulls in the testing set and their December 2011 DRP. Sire-maternal grandsire-maternal greatgrand sire index for sires in the testing set was used as a benchmark. It was calculated as $50 \%$ of the sire DRP, $25 \%$ of the maternal grand sire DRP, and $12.5 \%$ of the maternal great-grand sire DRP. For simplicity we refer to these values as sire-pedigree index (Sire-PI). Estimated accuracies were adjusted by EDC following the recommendations of Mäntysaari et al. (2010).

The average difference between 2011 DRP and the predicted DGV in the testing population provided a measure of bias in the genomic predictions; this bias estimate was standardized. Coefficients of regression of realized December 2011 DRP on estimated DGV were also calculated, because this parameter is also commonly used as a measure of prediction bias in genomic evaluations (Mäntysaari et al., 2010). Finally, MSE of prediction, which is linked to bias, slope, and accuracy, was also estimated.

\section{RESULTS AND DISCUSSION}

\section{Summary of Genomic Data}

The distribution of genotyped bulls, by year of birth, is shown in Figure 1. Most of the bulls were born af- ter 1990, providing a recent reference population for prediction of genomic values of young animals. It is well known that GS results in higher responses for the generations closer to the reference population (Goddard, 2009).

After filtering, the distribution of MAF was nearly uniform with a mean of 0.28 (Figure 2). The average distance between adjacent SNP was $0.06 \mathrm{Mb}$, and SNP had average heterozygosity of 0.29 . Linkage disequilibrium between adjacent SNP, measured as $r^{2}$, was 0.24 . All values were in the range reported previously values for other Holstein populations (Wiggans et al., 2009; Banos and Coffey, 2010; Habier et al., 2010).

Figure 3 shows the average $r^{2}$ between SNP pairs plotted against the map distance of up to $1 \mathrm{Mb}$ and shows the standard deviations for the average $r^{2}$ values across all 30 chromosomes. Average $r^{2}$ decreased exponentially with increasing distance between SNP and was equal to $0.40,0.24,0.16$, and 0.08 at distances of $0.01,0.05,0.1$, and $1 \mathrm{Mb}$, respectively. The level of decay in LD with respect to the distance between SNP was also similar to results from other populations (de Roos et al., 2009; Habier et al., 2010).

\section{Accuracy}

Traditional Sire-PI accuracies ranged from 0.37 to 0.51 (Table 1). Predicted DGV showed higher accuracies than Sire-PI, regardless of the genomic prediction model, with an average increment of $49 \%$, ranging from $9 \%$ for UD to $83 \%$ for FP. Similar results have been reported previously in other Holstein populations (VanRaden et al., 2009; Moser et al., 2010), indicating that selection of young animals based on genomic pre- 


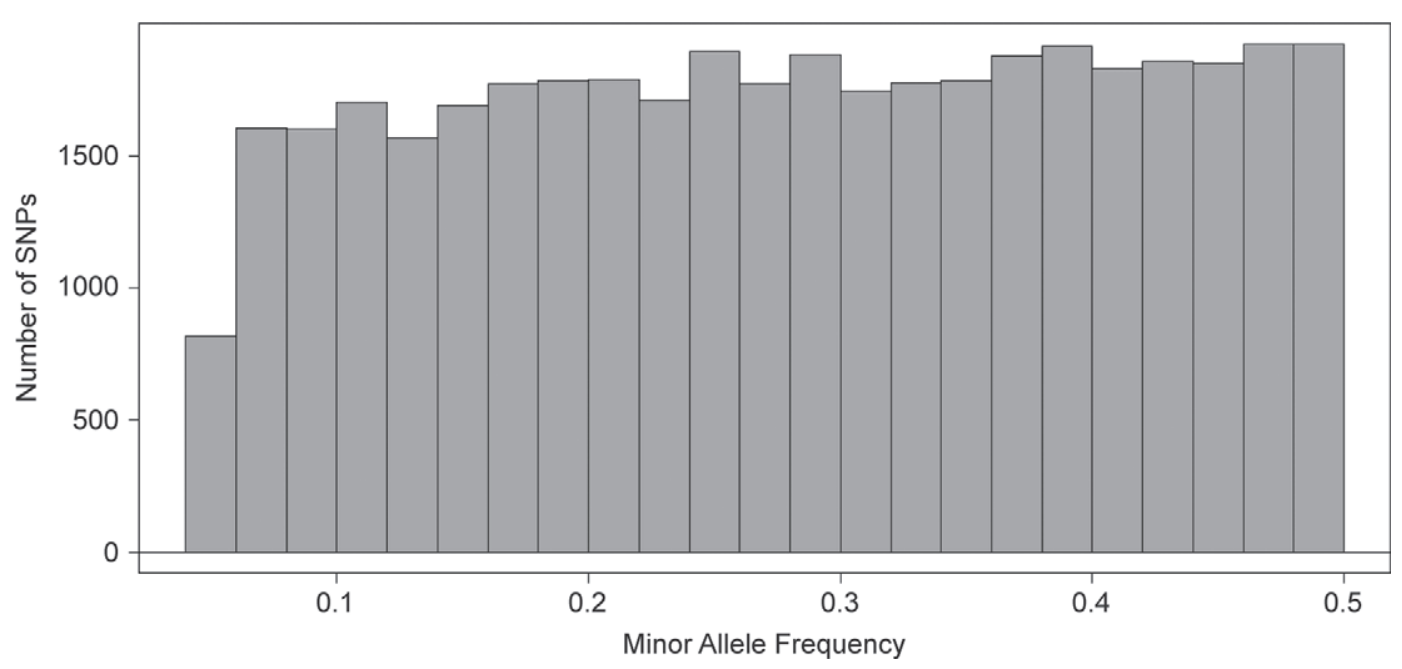

Figure 2. Distribution of minor allele frequencies (MAF) of the SNP after quality control.

dictions is preferable to selection based on traditional pedigree information.

Among methods, B-LASSO provided the highest Pearson correlations for MY (0.60), FY (0.61), and PY (0.57), as well as the highest Pearson correlation averaged across traits. Bayes-A provided greater accuracy for UD (0.56) and was equivalent in accuracy to BLASSO for FY (0.61). The R-Boost model achieved the greatest Pearson correlation for FP (0.81), whereas G-BLUP achieved the same accuracy as B-LASSO for PY (0.57). In general, differences in accuracy between methods were small for MY, FY, PY, and UD, but larger differences were found for FP. For instance, RBoost outperformed GBLUP by 0.19 units of Pearson correlation for FP. Pearson correlation coefficients averaged across traits were $0.61,0.60,0.58$, and 0.58 for BLASSO, R-Boost, Bayes-A, and G-BLUP, respectively.

In a previous study based on simulated and growth data in mice, Usai et al. (2009) showed slightly greater accuracy with B-LASSO compared with G-BLUP and Bayes-A. Cleveland et al. (2010) reported a similar predictive ability for B-LASSO and 2 variants of Bayes-A in simulated data; however, the authors observed better performance of B-LASSO for traits that were regulated by many QTL with small effects. Legarra et al. (2011) reported slightly greater accuracies for B-LASSO than for G-BLUP, but slightly better for B-LASSO. Genomic BLUP on real data showed reliabilities of $63 \%$ com-

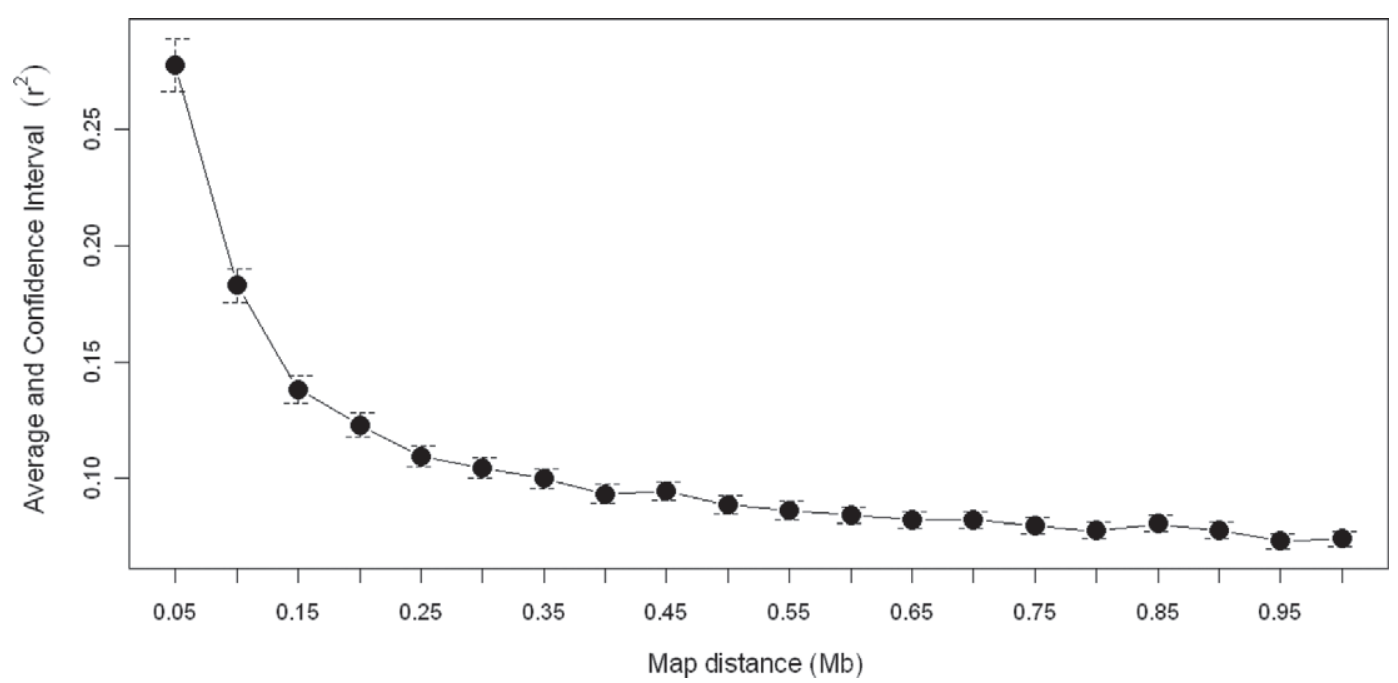

Figure 3. Average linkage disequilibrium (measured as $r^{2}$ ) and confidence interval (estimated by R package gplots; R Development Core Team, 2011) between synthenic markers with respect to their physical distance. 
Table 1.Accuracy, standardized bias in means, bias in regression coefficients, and mean squared error (MSE) of genomic predictions for different evaluation methodologies and 5 traits of economic interest in Spanish dairy cattle

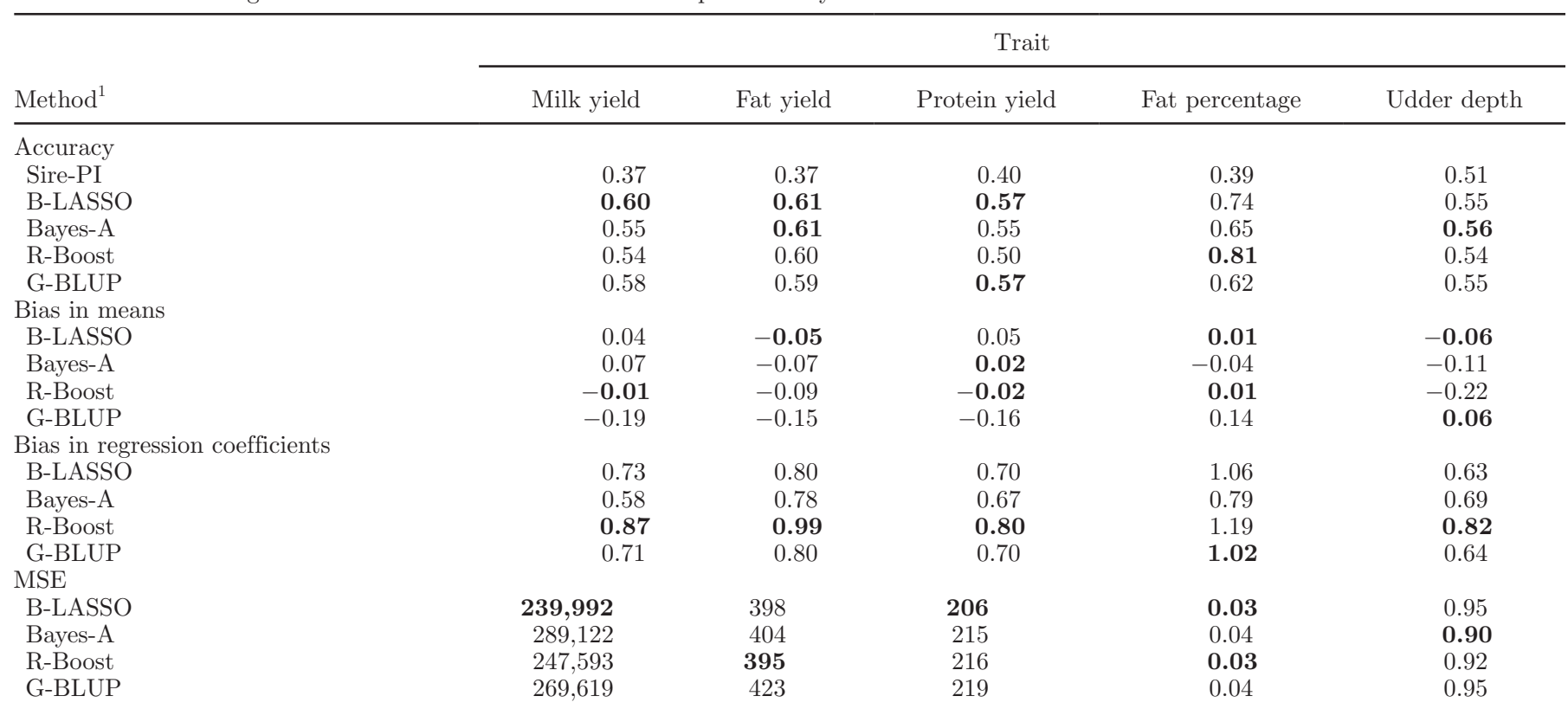

${ }^{1}$ Sire-PI $=$ traditional pedigree index; B-LASSO = Bayesian least absolute shrinkage selection operator; R-Boost = random boosting; and G-BLUP = genomic BLUP. The preferred method within trait and comparison criteria is shown in bold.

pared with $32 \%$ from pedigree index on the combined trait net merit (VanRaden, 2008). Others found higher or similar accuracies using GBLUP than using Bayes-B (Luan et al., 2009; Mrode et al., 2010).

We found no relevant differences between R-Boost and the additive models based on marker regression, except for FP. Although machine learning techniques are expected to accommodate cryptic relationships in the data, the use of dependent variables that represent previously computed (additive, linear and smoothed) sire EBV could mask such differences. The R-Boost method seems to provide some advantages over Bayesian regression when a small number of QTL regulate the trait under purely additive regulation (GonzálezRecio and Forni, 2011). In the present study, genomic predictions from R-Boost were more accurate for traits controlled by single genes that explain a large proportion of the genetic variance (e.g., DGAT1 for FP). Note that differences exist in accuracy for the R-Boost method between the results of this and the companion paper (González-Recio et al., 2013), presumably due to the adjustment for number of progeny in the present paper as suggested by Mäntysaari et al. (2010).

\section{Bias in the Mean}

The DGV of bulls in the testing set showed an average deviation over the realized DRP of 0.08 genetic
SD across methods and traits, with averages ranging from 0.05 (FP) to 0.11 (UD). Increasing the size of the reference population may alleviate this problem (Liu et al., 2011; Lund et al., 2011). Standardized bias showed greater differences between methods than Pearson correlations. The R-Boost model resulted in nearly unbiased predictions for MY and FP and also produced the least bias for PY, whereas B-LASSO produced the least bias in predictions for FY, FP, and UD. BayesA showed a similar bias to R-Boost for PY. Genomic BLUP tended to provide more biased predictions for all traits, with the exception of UD. The methods with greater Pearson correlation can also produce more biased predictions, so both accuracy and bias should be considered when deciding which method has greater predictive ability. Therefore, MSE may be a more appropriate comparison criterion than Pearson correlation, as it combines accuracy and bias.

When the genomic predictions of young bulls are compared with highly reliable, progeny-tested bulls, biases from genomic predictions must be taken into account. In addition, genomic predictions of future performance are expected to be biased when only genomically preselected bulls are allowed to produce offspring (Patry and Ducrocq, 2011). This was not the case for bulls included in the present study, as they were genotyped after selection. 


\section{Bias in Regression Coefficients}

The coefficients of regressing realized DRP on estimated DGV are commonly used as a measure of bias in genomic evaluations. The expected value for this slope coefficient is unity if evaluations predict the actual magnitude of differences between bulls, if the genotyped young bulls are a representative sample of the bulls in the population. However, the genotyped young bulls are typically preselected by AI centers based on their EBV or Sire-PI (Mäntysaari et al., 2010). In our study, regression coefficients ranged between 0.58 for Bayes-A (MY) and 1.19 for the R-Boost (FP). The RBoost model provided the slope coefficient closest to unity for 4 of the 5 traits ( 0.87 for MY, 0.99 for FY, 0.80 for PY, and 0.82 for UD). Bayes-A provided the smallest coefficients for all traits, except UD, whereas B-LASSO and GBLUP produced similar coefficients that exceeded unity only for FP.

These regression coefficients were within the range reported in other studies in similar dairy cattle populations (Olson et al., 2011; Tsuruta et al., 2011). Some authors have suggested inclusion of a polygenic effect to address this problem (Liu et al., 2011), because this modification could reduce the overestimation of DGV. Low coefficients of regression for MY, PY, and UD could be explained by higher selection on these traits compared with FY and FP.

\section{MSE}

The MSE can be viewed as a risk function that incorporates both the predictive variance and bias of an estimator. The B-LASSO and R-Boost models provided the smallest MSE for all traits except UD, where Bayes-A outperformed the other methods. For instance, regarding MY, Bayes-A showed 20 and 17\% greater MSE than B-LASSO and R-Boost, respectively. Genomic BLUP also showed greater MSE (from 5 to $12 \%$ ) for MY and FY, compared with B-LASSO and R-Boost. The RBoost method was preferred across traits in terms of MSE, providing the smallest MSE on average, followed by B-LASSO, Bayes-A, and G-BLUP.

In a previous study, Verbyla et al. (2009) showed similar differences in MSE between Bayesian regression models and G-BLUP. Their study reported larger MSE than the present results for the Spanish population, perhaps due to their smaller reference population (1,098 progeny-tested bulls). As stated previously, MSE reflects both bias and accuracy but it is often ignored when comparing genomic evaluation methods.

\section{CONCLUSIONS}

Implementation of GS in the Spanish Holstein breeding program will improve selection efficiency for both
AI centers and commercial farms, and identification of superior animals at a young age will be more accurate than was previously thought possible. The descriptors of the genomic structure of the population used in this study showed that the Spanish population is similar to other Holstein dairy cattle populations, as expected. Based on this similarity, genomic evaluations of genotyped animals for recorded traits included in the milk recording scheme should be feasible. Different prediction methodologies (including nonparametric methods) implemented in this study showed similar predictive ability, and the optimal method was sometimes trait dependent. In general, B-LASSO was preferable in terms of Pearson correlations, and R-Boost provided regression coefficient estimates closest to unity. Both methods outperformed Bayes-A and G-BLUP in terms of predicted MSE. Methods that provided higher Pearson correlations also showed large biases, so MSE may be a more appropriate comparison criterion than Pearson correlations. Marker regression methods outperformed G-BLUP in terms of MSE because of larger bias in G-BLUP estimates. Future collaborations with the Eurogenomics consortium, which has a reference population of more than 20,000 progeny-tested bulls, are expected to substantially increase the accuracy of genomic predictions for Spanish Holsteins. Here, the RBoost method is expected to show some computational advantages over B-LASSO and Bayes-A.

\section{ACKNOWLEDGMENTS}

The authors acknowledge funds from the project CDTI-P080250866 UPM and the agreement INIACC10-046, to CONAFE, ASCOL, ABEREKIN, XENETICA FONTAO and GENETICAL for providing biological samples and phenotypes used in this study and to "Dirección General de Producciones y Mercados Agrarios," "Laboratorio Central de Veterinaria del Ministerio de Agricultura, Alimentación y Medio Ambiente" for support of genotyping process and especially Kent Weigel for helpful suggestions and comments.

\section{REFERENCES}

Aguilar, I., I. Misztal, D. L. Johnson, A. Legarra, S. Tsuruta, and T. J. Lawlor. 2010. A unified approach to utilize phenotypic, full pedigree, and genomic information for genetic evaluation of Holstein final score. J. Dairy Sci. 93:743-752.

Banos, G., and M. P. Coffey. 2010. Short communication: Characterization of the genome-wide linkage disequilibrium in 2 divergent selection lines of dairy cows. J. Dairy Sci. 93:2775-2778.

Browning, B. L., and S. R. Browning. 2009. A unified approach to genotype imputation and haplotype phase inference for large data sets of trios and unrelated individuals. Am. J. Hum. Genet. $84: 210-223$.

Cleveland, M. A., S. Forni, N. Deeb, and C. Maltecca. 2010. Genomic breeding value prediction using three Bayesian methods and ap- 
plication to reduced density marker panels. BMC Proc. 4(Suppl. 1):S6.

Daetwyler, H. D., R. Pong-Wong, B. Villanueva, and J. A. Woolliams. 2010. The impact of genetic architecture on genome-wide evaluation methods. Genetics 185:1021-1031.

de los Campos, G., D. Gianola, and D. B. Allison. 2010. Predicting genetic predisposition in humans: The promise of whole-genome markers. Nat. Rev. Genet. 11:880-886.

de los Campos, G., H. Naya, D. Gianola, J. Crossa, A. Legarra, E. Manfredi, K. Weigel, and J. M. Cotes. 2009. Posterior predicting quantitative traits with regression models for dense molecular markers and pedigrees. Genetics 182:375-385.

de Roos, A. P., B. J. Hayes, and M. E. Goddard. 2009. Reliability of genomic predictions across multiple populations. Genetics 183:1545-1553.

Friedman, J. H. 2001. Greedy function approximation: A gradient boosting machine. Ann. Stat. 29:1189-1232.

Gianola, D., G. A. de los Campos, W. G. Hill, E. Manfredi, and R. L. Fernando. 2009. Additive genetic variability and the Bayesian alphabet. Genetics 183:347-363.

Gianola, D., and J. B. C. H. M. van Kaam. 2008. Reproducing kernel Hilbert spaces regression methods for genomic assisted prediction of quantitative traits. Genetics 178:2289-2303.

Goddard, M. 2009. Genomic selection: Prediction of accuracy and maximisation of long term response. Genetica 136:245-257.

González-Recio, O., and S. Forni. 2011. Genome-wide prediction of discrete traits using Bayesian regressions and machine learning. Genet. Sel. Evol. 43:7.

González-Recio, O., D. Gianola, N. Long, K. A. Weigel, G. J. M. Rosa, and S. Avendaño. 2008. Nonparametric methods for incorporating genomic information into genetic evaluations: An application to mortality in broilers. Genetics 178:2305-2313.

González-Recio, O., D. Gianola, G. J. Rosa, K. A. Weigel, and A. Kranis. 2009. Genome-assisted prediction of a quantitative trait measured in parents and progeny: Application to food conversion rate in chickens. Genet. Sel. Evol. 41:3.

González-Recio, O., J. A. Jiménez-Montero, and R. Alenda. 2013. The gradient boosting algorithm and random boosting for genomicassisted evaluation in large data sets. J. Dairy Sci. 96:614-624. http://dx.doi.org/10.3168/jds.2012-5630.

González-Recio, O., K. A. Weigel, D. Gianola, H. Naya, and G. J. M. Rosa. 2010. L2-Boosting algorithm applied to high-dimensional problems in genomic selection. Genet. Res. (Camb.) 92:227-237.

Habier, D., J. Tetens, F. R. Seefried, P. Lichtner, and G. Thaller. 2010. The impact of genetic relationship information on genomic breeding values in German Holstein cattle. Genet. Sel. Evol. 42:5.

Hastie, T. J., R. Tibshirani, and J. Friedman. 2009. The Elements of Statistical Learning. 2nd ed. Springer, New York, NY.

Hayes, B. J., P. J. Bowman, A. J. Chamberlain, and M. E. Goddard. 2009a. Genomic selection in dairy cattle: Progress and challenges. J. Dairy Sci. 92:433-443.

Hayes, B. J., P. J. Bowman, A. J. Chamberlain, K. Savin, C. P. van Tassell, T. S. Sonstegard, and M. E. Goddard. 2009b. A validated genomewide association study to breed cattle adapted to an environment altered by climate change. PLoS ONE 4:e6676. http:// dx.doi.org/10.1371/journal.pone.0006676.

Hill, W. G., and A. Robertson. 1968. Linkage disequilibrium in finite populations. Theor. Appl. Genet. 38:226-231.

Jairath, L., J. C. M. Dekkers, L. R. Schaeffer, Z. Liu, E. B. Burnside, and B. Kolstad. 1998. Genetic evaluation of herd life in Canada. J. Dairy Sci. 81:550-562.

Legarra, A., C. Robert-Granié, P. Croiseau, F. Guillaume, and S. Fritz. 2011. Improved LASSO for genomic selection. Genet. Res. (Camb.) 93:77-87.

Liu, Z., F. R. Seefried, F. Reinhardt, S. Rensing, G. Thaller, and R. Reents. 2011. Impacts of both reference population size and inclusion of a residual polygenic effect on the accuracy of genomic prediction. Genet. Sel. Evol. 43:19.

Long, N., D. Gianola, G. J. M. Rosa, S. Weigel, and S. Avendano. 2007. Machine learning classification procedure for selecting SNPs in genomic selection: Application to early mortality in broilers. J. Anim. Breed. Genet. 124:377-389.

Luan, T., J. A. Woolliams, S. Lien, M. Kent, M. Svendsen, and T. H. E. Meuwissen. 2009. The accuracy of genomic selection in Norwegian Red cattle assessed by cross validation. Genetics 183:1119-1126.

Lund, M. S., S. P. W. de Ross, A. G. de Vries, T. Druet, V. Ducrocq, S. Fritz, F. Guillaume, B. Guldbrandtsen, Z. Liu, R. Reents, C. Schrooten, F. Seefried, and G. Su. 2011. A common reference population from four European Holstein populations increases reliability of genomic predictions. Genet. Sel. Evol. 43:43.

Mäntysaari, E., Z. Liu, and P. M. VanRaden. 2010. Interbull validation test for genomic evaluations. Interbull Bull. 41:17-22.

Meuwissen, T. H. E., B. J. Hayes, and M. E. Goddard. 2001. Prediction of total genetic value using genome-wide dense marker maps. Genetics 157:1819-1829.

Misztal, I., A. Legarra, and I. Aguilar. 2009. Computing procedures for genetic evaluation including phenotypic, full pedigree, and genomic information. J. Dairy Sci. 92:4648-4655.

Moser, G., M. S. Khatkar, B. Hayes, and H. W. Raadsma. 2010. Accuracy of direct genomic values in Holstein bulls and cows using subsets of SNP markers. Genet. Sel. Evol. 42:37.

Moser, G., B. Tier, R. E. Crump, M. S. Khatkar, and H. W. Raadsma. 2009. A comparison of five methods to predict genomic breeding values of dairy bulls from genome-wide SNP markers. Genet. Sel. Evol. 41:56.

Mrode, R., M. P. Coffey, I. Strandén, T. H. E. Meuwissen, J. B. C. H. M. Van Kaam, J. F. Kearney, and D. P. Berry. 2010. A comparison of various methods for the computation of genomic breeding values of dairy bulls using software at genomicselection.net. Abstract 518 in 9th World Congress on Genetics Applied to Livestock Production, Leipzig, Germany.

Ogutu, J. O., H. P. Piepho, and T. Schulz-Streeck. 2011. A comparison of random forests, boosting and support vector machines for genomic selection. BMC Proc. 5(Suppl. 3):S11.

Olson, K. M., P. M. VanRaden, M. E. Tooker, and T. A. Cooper. 2011. Differences among methods to validate genomic evaluations for dairy cattle. J. Dairy Sci. 94:2613-2620.

Park, T., and G. Casella. 2008. The Bayesian LASSO. J. Am. Stat. Soc. 103:681-686.

Patry, C., and V. Ducrocq. 2011. Evidence of biases in genetic evaluations due to genomic preselection in dairy cattle. J. Dairy Sci. 94:1011-1020.

Pryce, J. E., J. Arias, P. J. Bowman, S. R. Davis, K. A. Macdonald, G. C. Waghorn, W. Wales, Y. J. Williams, R. J. Spelman, and B. J. Hayes. 2012. Accuracy of genomic predictions of residual feed intake and 250 day body weight in growing heifers using 625000 SNP markers. J. Dairy Sci. 95:2108-2119.

R Development Core Team. 2011. R: A language and environment for statistical computing. R Foundation for Statistical Computing, Vienna, Austria. http://www.R-project.org/.

Sargolzaei, M., F. S. Schenkel, J. B. Jansen, and L. R. Schaeffer. 2008. Extent of linkage disequilibrium in Holstein cattle in North America. J. Dairy Sci. 91:2106-2117.

Tibshirani, R. 1996. Regression shrinkage and selection via the LASSO. J. R. Stat. Soc. Ser. B Methodol. 58:267-288.

Toosi, A., R. L. Fernando, and J. C. M. Dekkers. 2010. Genomic selection in admixed and crossbred populations. J. Anim. Sci. $88: 32-46$

Tsuruta, S., I. Misztal, I. Aguilar, and T. J. Lawlor. 2011. Multiple trait genomic evaluation of linear type traits using genomic and phenotypic data in US Holsteins. J. Dairy Sci. 94:4198-4204.

Usai, M. G., M. E. Goddard, and B. J. Hayes. 2009. LASSO with cross-validation for genomic selection. Genet. Res. (Camb.) 91:427-436.

VanRaden, P. M. 2008. Efficient methods to compute genomic predictions. J. Dairy Sci. 91:4414-4423.

VanRaden, P. M., and P. G. Sullivan. 2010. International genomic evaluation methods for dairy cattle. Genet. Sel. Evol. 42:7.

VanRaden, P. M., C. P. Van Tassell, G. R. Wiggans, T. S. G. Sontegard, R. D. Schnabel, J. F. Taylor, and F. S. Schenkel. 2009. Reli- 
ability of genomic predictions for North American Holstein bulls. J. Dairy Sci. 92:16-24.

Verbyla, K. L., B. J. Hayes, P. J. Bowman, and M. E. Goddard. 2009. Accuracy of genomic selection using stochastic variable selection in Australian Holstein Friesian dairy cattle. Genet. Res. (Camb.) 91:307-311.

Weigel, K. A., P. C. Hoffman, W. Herring, and T. J. Lawlor Jr. 2012. Potential gains in lifetime net merit from genomic testing of cows, heifers, and calves on commercial dairy farms. J. Dairy Sci. 95:2215-2225.

Weigel, K. A., C. P. Van Tassell, J. R. O'Connel, P. M. VanRaden, and G. R. Wiggans. 2010. Prediction of unobserved single nucleotide polymorphism genotypes of Jersey cattle using reference panels and population-based imputation algorithms. J. Dairy Sci 93:2229-2238.
Wiggans, G. R., T. S. Sonstegard, P. M. VanRaden, L. K. Matukumalli, R. D. Schnabel, J. F. Taylor, F. S. Schenkel, and C. P. Van Tassell. 2009. Selection of single-nucleotide polymorphisms and quality of genotypes used in genomic evaluation of dairy cattle in the United States and Canada. J. Dairy Sci. 92:3431-3436.

Wiggans, G. R., P. M. VanRaden, and T. A. Cooper. 2011. The genomic evaluation system in the United States: Past, present, future. J. Dairy Sci. 94:3202-3211.

Yi, N. J., and S. H. Xu. 2008. Bayesian LASSO for quantitative trait loci mapping. Genetics 179:1045-1055.

Zhao, H., D. Nettleton, M. Soller, and J. C. M. Dekkers. 2005. Evaluation of linkage disequilibrium measures between multi-allelic markers as predictors of linkage disequilibrium between markers and QTL. Genet. Res. (Camb.) 86:77-87. 